\title{
CANAGLIFLOZIN REDUCES THE RISK OF KIDNEY \\ FAILURE IN PATIENTS WITH TYPE 2 DIABETES \\ MELLITUS AND NEPHROPATHY: THE CREDENCE RANDOMIZED TRIAL
}

QUALITY OF EVIDENCE: HIGH

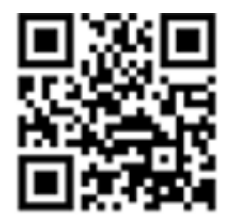

Want to learn more? Check out the SGIM Bottom Line Podcast on iTunes, Spotify, or wherever you get your podcasts. Go to sgimbottomline.com or scan the QR code for details.

\section{THE BOTTOM LINE}

In patients with type 2 diabetes mellitus and nephropathy, canagliflozin $100 \mathrm{mg}$ daily reduced the risk of kidney failure when compared with placebo over a mean follow up of 2.6 years.

\section{Why is This Important?}

- The global prevalence of end-stage kidney disease is increasing, mainly because of the increase in the number of patients diagnosed with diabetes mellitus (DM). Currently, more than 3 million people worldwide are receiving treatment for kidney failure, and it is predicted that this number will increase to more than 5 million people by 2035 . [1, 2]

- The only currently approved treatment for renoprotection in patients with type 2 diabetes is renin-angiotensin system blockade, which was first shown to be effective 18 years ago. [2]

- Previous studies suggested a trend for patients with type 2 diabetes and chronic kidney disease in that the use of a sodium-glucose cotransporter 2 (SGLT2) inhibitor reduces the risk of chronic kidney disease progression [3], but none of the previous studies were powered to show a difference.

\section{Facts}

- CREDENCE [4] is a randomized, double blind, placebo-controlled, multicenter trial. A total of 4401 patients with type 2 diabetes and albuminuric chronic kidney disease (urine microalbumin to creatinine ratio $>300 \mathrm{mg} / \mathrm{g}$, eGFR $30-90 \mathrm{ml} / \mathrm{min} / 1.73 \mathrm{~m}$ ) on maximum tolerated dose of ACEI or ARBs were randomized to receive $100 \mathrm{mg}$ canagliflozin (an oral SGLT2 inhibitor) daily or placebo and were followed for 2.62 years. The average age of participants was 63 years, $33.9 \%$ females, 66.6\% white, mean duration T2DM 15.8 years, and $\mathrm{HgA} 1 \mathrm{c} 8.3 \%$, with mean baseline eGFR $56.2 \mathrm{ml} / \mathrm{min} / 1.73 \mathrm{~m}$.

- Patients with type 1 diabetes, immunosuppression for kidney disease, or a history of dialysis or kidney transplant were excluded. 
Doubling of serum creatinine level, end-stage kidney disease, renal death or cardiovascular death

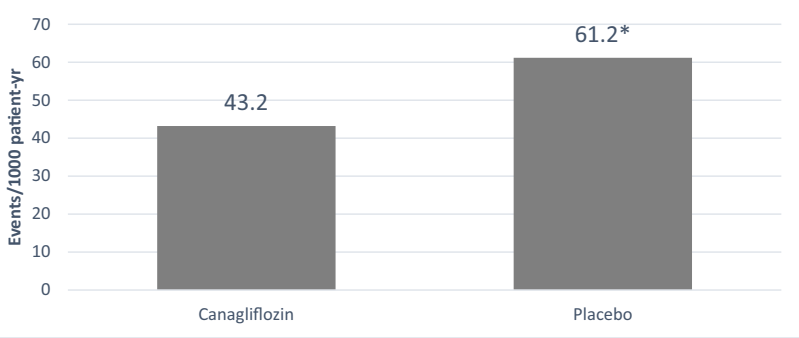

Fig. 1 Primary composite outcome

- The primary composite outcome (doubling of serum creatinine level, end-stage kidney disease, renal death, or cardiovascular death) was significantly lower in the canagliflozin group than in the placebo group (HR $0.70 ; 95 \% \mathrm{Cl} 0.59$ to $0.82, p=$ $0.00001) \mathrm{NNT}=22$ over 2.62 years $(95 \% \mathrm{Cl} 15-38)$ (see Fig. 1). Other secondary outcomes, including a composite cardiovascular event outcome, renal outcomes, and hospitalizations for heart failure, were all significantly less frequent in the canagliflozin group.

- The trial was stopped early for benefit at a planned interim analysis, after 2.62 years of follow-up out of 5.5 years planned.
- Subgroup analysis showed no significant difference based on patients' GFR or albumin-tocreatinine ratio at baseline

- Amputation rates were similar between the groups, with 12.3 versus 11.2 events/1000 person years in the canagliflozin group versus the placebo group. CANVAS-R [3] previously showed increased risk of amputations with 6.3 events in the canagliflozin group versus 3.4 events/1000 patient-years in the placebo group.

\section{Study Quality and Applicability Considerations}

Study internal validity was preserved because of the randomization concealment and blinding of patients and clinicians. Both groups received the same follow-up visits and treatments which minimized performance bias or any other bias that could have potentially been introduced during the study period. The trial was stopped early for benefit based on predefined criteria when the sample size was already achieved. There was low risk of bias potentially introduced by stopping the trial early for benefit. The external validity of the study is preserved as it was a multicenter trial. 


\section{TIPS FOR DISCUSSION WITH PATIENTS}

- Canagliflozin taken daily by patients with type 2 diabetes and chronic kidney disease can decrease the risk of kidney failure and cardiovascular events like heart attack, stroke, or death.

- Patients already prescribed maximum tolerated doses of ACEl/ARB received additional benefit from canagliflozin regardless of their kidney function at baseline.

- Rates of side effects were similar between canagliflozin and a placebo, or sugar pill, in this trial.

\section{AUTHORS}

Corresponding Author: Alexandra Halalau, MD; Division of General Internal Medicine,

Department of Medicine Beaumont Hospital, Royal Oak, MI, USA

(e-mail: Alexandra.halalau@beaumont.edu).
Alexandra Halalau, MD

William Fuller, MD

Stephanie Wheeler, Md, MPH

Compliance with Ethical Standards: The authors have no conflict of interest to disclose.

\section{References}

1. International Diabetes Federation. IDF diabetes atlas. 8th ed. Brussels: International Diabetes Federation, 2017

2. Liyanage $T$, Ninomiya $T$, Jha $\mathbf{V}$, et al. Worldwide access to treatment for end- stage kidney disease: a systematic review. Lancet 2015;385:1975-1982

3. Neal B, Perkovic V, Mahaffey KW, et al. Canagliflozin and cardiovascular and renal events in type 2 diabetes. $\mathrm{N}$ Engl J Med 2017;377:644-657
4. Perkovic V, Jardine M.J., Neal B, et al. Canagliflozin and renal outcomes in type 2 diabetes and nephropathy. N Engl J Med 2019; 380:2295-2306

Publisher's Note: Springer Nature remains neutral with regard to jurisdictional claims in published maps and institutional affiliations.

The Bottom Line summaries reflect the expertise and opinions of the SGIM EBM Task Force as of the date of release of this summary.

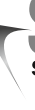

